\title{
Will new isotope sources be ready in time?
}

\section{$\mathrm{W}$} ith the National Reactor Universal (NRU) in Chalk River, Ontario, ceasing production of medical isotopes in October 2016, Canada risks losing a major medical industry that has supplied $40 \%$ $50 \%$ of the world's molybdenum-99 (Mo-99) for imaging and $85 \%$ of the world's cobalt-60 for radiotherapy. New sources and approaches are scaling up quickly to fill the gap, but in most cases, production volumes and timelines have not yet been established. As one expert told CMAJ, "It's very difficult to project what is going to happen."

Canadian isotope extraction and purification company Nordion will source Mo-99 from the University of Missouri Research Reactor beginning in 2017, according to a news release. Nordion already uses cobalt-60 from Bruce Power in Tiverton, Ontario, for sterilization of medical equipment and supplies. Bruce Power also plans to produce medical-grade cobalt-60 for radiotherapy, although details of the technology and timeline were not provided.

A promising new way to replace the NRU's Mo-99 involves cyclotrons, a particle accelerator often used in hospitals to produce isotopes such as fluorine-18 for positron-emission tomography. Cyclotrons can also produce technetium-99m (Tc-99m), the final product of Mo-99, but this has never been done on a commercial scale. In fact, the first successful commercial-scale demonstration was a little over a year ago, when enough Tc-99m to fulfill a day's demand in British Columbia was produced in a six-hour run using a common medical cyclotron at TRIUMF, a particle and nuclear physics laboratory in Vancouver run by a group of Canadian universities.

"Our philosophy all along has been to enable our existing Canadian infrastructure," said Paul Schaffer, associate lab director of TRIUMF's Life Science Division and head of the project. "Canada has one of the world's most advanced fleet of cyclotrons in hospitals and research institutions. This provides very significant production capacity."

There are 24 medical cyclotrons in 12 cities across Canada; 20 can produce Tc-99m. The plan is for 11 of these



Paul Schaffer, head of the cyclotron project in British Columbia, peers into a "hot cell."

cyclotrons to eventually produce Tc$99 \mathrm{~m}$, to meet much of the demand in the centres where they are located. Schaffer estimates that each facility could be brought up to production within about eight months, but target dates are not yet established for most facilities.

Cyclotron-produced Tc-99m differs from reactor-produced Mo-99. For one thing, it has a half-life of only six hours. "There's no way it can serve the whole country," said Dr. Andrew Ross, president of the Canadian Association of Nuclear Medicine. Schaffer says centres not served by cyclotrons, and rural areas, would need to obtain Mo-99 or Tc-99m from other sources.

Full commercial production could be substantially delayed while the industry meets regulatory requirements from the Canadian Nuclear Safety Commission and Health Canada, said Ross. "It is a very long and arduous process."

Schaffer said his team has worked closely with Health Canada at all steps in the regulatory approval process, which includes a clinical trial. Dr. François Bénard, who works for the functional imaging department of the $\mathrm{BC}$ Cancer Agency, is heading that trial, which includes some 60 patients at two sites who require thyroid gland and bone scans.

"One indication addresses the biological function of the product," Bénard said, and the other "allows us to measure any differences in image quality in cyclotron-produced versus reactor-produced technetium." The trial is expected to conclude this spring and Schaffer anticipates Health Canada approval in late 2016 or early 2017.

Each cyclotron facility in Canada needs separate approval, said Schaffer, but by sharing the regulatory paperwork, the time to approval can be significantly reduced.

Another hurdle is each province's procurement process; individual provincial governments arrange purchases from producers. Discussions with the BC government are currently underway, Schaffer said. "We can't wait for regulatory approval."

The TRIUMF-led cyclotron consortium is among several cyclotron and linear accelerator isotope solutions that have received $\$ 60$ million from the federal government from 2010-2016.

Schaffer described the capital funding as a "mosaic of investment" from various players. A final investment is needed to construct a building for a recently acquired cyclotron to help TRIUMF meet demand in BC. That cyclotron facility will have a final price tag of about \$30 million; other cyclotrons cost \$10-\$15 million depending on size and configuration. "The cost is still relatively small compared to running a reactor," said Schaffer. - Carolyn Brown, Ottawa, Ont.

CMAJ 2016. DOI:10.1503/cmaj.109-5224 